\title{
Defect Model of a Tetragonal $\mathrm{Sm}^{3+}$ Center Found from EPR Measurements in $\mathrm{CaF}_{2}$ and $\mathrm{SrF}_{2}$ Crystals
}

\author{
Wen-Chen Zheng ${ }^{\mathrm{a}, \mathrm{b}}$, Hui-Ning Dong ${ }^{\mathrm{a}, \mathrm{c}}$, Shao-Yi Wu ${ }^{\mathrm{b}, \mathrm{d}}$, and Sheng Tang ${ }^{\mathrm{a}}$ \\ ${ }^{a}$ Department of Material Science, Sichuan University, Chengdu 610064, P. R. China \\ ${ }^{\mathrm{b}}$ International Centre for Materials Physics, Chinese Academy of Sciences, \\ Shenyang 110016, P. R. China \\ ${ }^{c}$ Institute of Solid State Physics, Sichuan Normal University, Chengdu 610066, China \\ d Department of Applied Physics, University of Electronic Science and Technology of China, \\ Chendu 610054, P. R. China
}

Reprint requests to W.-C. Z.; E-mail: zhengwenchen@ netease.com

Z. Naturforsch. 58a, 373 - 375 (2003); received February 14, 2003

The EPR parameters ( $\mathrm{g}$ factors $g_{\|}, g_{\perp}$ and hyperfine structure constants $A_{\|}, A_{\perp}$ ) of a tetragonal $\left(\mathrm{C}_{4 \mathrm{v}}\right) \mathrm{Sm}^{3+}$ center in $\mathrm{CaF}_{2}$ and $\mathrm{SrF}_{2}$ crystals are calculated by considering the crystal-field $\mathrm{J}$ mixing among the ground ${ }^{6} \mathrm{H}_{5 / 2}$, the first excited ${ }^{6} \mathrm{H}_{7 / 2}$ and second excited ${ }^{6} \mathrm{H}_{9 / 2}$ state multiplets. In the calculations the free-ion and crystal-field parameters of the tetragonal $\mathrm{Sm}^{3+}-\mathrm{F}^{-}$center obtained from polarized laser-selective excitation spectroscopy are used. The calculated results suggest that the tetragonal $\mathrm{Sm}^{3+}-\mathrm{F}^{-}$center is the $\mathrm{Sm}^{3+}$ center found by later EPR measurements. The $\mathbf{g}$ factors $g_{\|}, g_{\perp}$ and hyperfine structure constants $A_{\|}, A_{\perp}$ of this EPR center are satisfactorily explained.

Key words: Defect Model; Electron Paramagnetic Resonance; Crystal-field Theory; $\mathrm{Sm}^{3+} ; \mathrm{CaF}_{2} ; \mathrm{SrF}_{2}$.

\section{Introduction}

The doping of alkaline earth fluorides $\mathrm{CaF}_{2}$ and $\mathrm{SrF}_{2}$ with rare earth ions usually results in the replacement of divalent alkaline ions by trivalent rare earth $\left(\mathrm{Re}^{3+}\right)$ ions. The required charge compensation can occur in many ways, leading to sites with cubic, trigonal, tetragonal or rhombic symmetry $[1,2]$. There may be different impurity centers with even a consistent axial $\left(\mathrm{C}_{4 \mathrm{v}}\right.$ or $\left.\mathrm{C}_{3 \mathrm{v}}\right)$ symmetry in $\mathrm{Re}^{3+}$-doped $\mathrm{CaF}_{2}$ and $\mathrm{SrF}_{2}$ crystals. For example, early EPR studies $[1,3]$ found a tetragonal $\left(\mathrm{C}_{4 \mathrm{v}}\right) \mathrm{Sm}^{3+}$ center with $g_{\|} \approx 0.907(10)$ and $g_{\perp} \approx 0.544(10)$ in $\mathrm{CaF}_{2}: \mathrm{Sm}^{3+}$. Weber and Bierig [1] suggested that this center probably arises from an interstitial $\mathrm{F}^{-}$charge compensator located at the center of one of the nearest empty cubes of the lattice (so we name it $\mathrm{Sm}^{3+}-\mathrm{F}^{-}\left(\mathrm{C}_{4 \mathrm{v}}\right)$ center). On later studies [4-7] of EPR in $\mathrm{CaF}_{2}: \mathrm{Sm}^{3+}$ and $\mathrm{SrF}_{2}: \mathrm{Sm}^{3+}$ a consistent $\mathrm{C}_{4 \mathrm{v}}$ symmetry center was found that was spectroscopically distinct (e.g., $g_{\|} \approx$ $0 \pm 0.06, g_{\perp} \approx 0.823 \pm 0.003$ in $\mathrm{CaF}_{2}: \mathrm{Sm}^{3+}$ ) and thus differed in the charge compensation configuration from the earlier studies. However, this $\mathrm{Sm}^{3+}\left(\mathrm{C}_{4 \mathrm{v}}\right)$ center was also suggested as $\mathrm{Sm}^{3+}{ }_{-} \mathrm{F}^{-}\left(\mathrm{C}_{4 \mathrm{v}}\right)$ center $[7,8]$. It is interest to determine which EPR center is the $\mathrm{Sm}^{3+}$ $\mathrm{F}^{-}\left(\mathrm{C}_{4 \mathrm{v}}\right)$ center. Polarized laser-selective excitation is a well-established method for identifying lines of multicenter spectra [9]. Polarized laser-selective excitation and fluorescence spectroscopy of $\mathrm{Sm}^{3+}$-doped $\mathrm{CaF}_{2}$ and $\mathrm{SrF}_{2}$ crystals was recently performed by Wells and Reeves [10]. They [10] found that the dominant center present in both host crystals is the $\mathrm{Sm}^{3+}-\mathrm{F}^{-}$ $\left(\mathrm{C}_{4 \mathrm{v}}\right)$ center and the optical spectral parameters (i.e., the free-ion and crystal-field parameters) of this center in both crystals were obtained (see Table 1). Although they pointed out that there are two different $\mathrm{Sm}^{3+}\left(\mathrm{C}_{4 \mathrm{v}}\right)$ centers found by the EPR measurements, they did not suggest which EPR $\mathrm{Sm}^{3+}\left(\mathrm{C}_{4 \mathrm{v}}\right)$ center is the $\mathrm{Sm}^{3+}-\mathrm{F}^{-}\left(\mathrm{C}_{4 \mathrm{v}}\right)$ center. Since the EPR parameters ( $\mathbf{g}$ factors and hyperfine structure constants $\mathrm{A}$ ) of a $4 \mathrm{f}^{n}$ ion in low symmetry are sensitive to the optical spectral parameters, in this paper we have calculated the EPR $\mathbf{g}$ factors $g_{\|}, g_{\perp}$ and hyperfine structure constants $A_{\|}, A_{\perp}$ for a tetragonal $\mathrm{Sm}^{3+}$ center in $\mathrm{CaF}_{2}$ and $\mathrm{SrF}_{2}$ crystals by using the above optical spectral parameters. The results (which are related to the assignment of the EPR $\mathrm{C}_{4 \mathrm{v}}$ center) are discussed. 
Table 1. Free-ion and crystal-field parameters (in $\mathrm{cm}^{-1}$ ) for the $\mathrm{Sm}^{3+}-\mathrm{F}^{-}\left(\mathrm{C}_{4 \mathrm{v}}\right)$ centers in $\mathrm{CaF}_{2}$ and $\mathrm{SrF}_{2}$ crystals [10].

\begin{tabular}{ccc}
\hline Parameter & $\mathrm{CaF}_{2}: \mathrm{Sm}^{3+}$ & $\mathrm{SrF}_{2}: \mathrm{Sm}^{3+}$ \\
\hline$F^{2}$ & 78824 & 79062 \\
$F^{4}$ & 56842 & 56870 \\
$F^{6}$ & 39972 & 40077 \\
$\alpha$ & 20.6 & 20.6 \\
$\beta$ & -724 & -724 \\
$r$ & 1700 & 1700 \\
$\zeta_{4 \mathrm{f}}$ & 1166 & 1168 \\
$B_{A}^{2}$ & 746 & 472 \\
$B_{A}^{4}$ & 590 & 545 \\
$B_{A}^{6}$ & 617 & 489 \\
$B_{C}^{2}$ & -1227 & -1204 \\
$B_{C}^{4}$ & 670 & 562 \\
\hline
\end{tabular}

\section{Calculations}

The ground state of a free $\mathrm{Sm}^{3+}\left(4 \mathrm{f}^{5}\right)$ ion is ${ }^{6} \mathrm{H}_{5 / 2}$, which is split into three Kramers doublets in a tetragonal crystal field. The lowest (or ground) doublet $\Gamma \gamma$ may be $\Gamma_{6}$ or $\Gamma_{7}$ depending upon the crystal field parameters. Since the $\mathbf{g}$ factors calculated by considering the mixing in only the ground state multiplet ${ }^{6} \mathrm{H}_{5 / 2}$ or, further, the crystal-field J-mixing of the first excited state multiplet ${ }^{6} \mathrm{H}_{7 / 2}$ into the ground state multiplet ${ }^{6} \mathrm{H}_{5 / 2}$ can not agree with the observed values [11], we consider the J-mixing among the ground ${ }^{6} \mathrm{H}_{5 / 2}$, the first excited ${ }^{6} \mathrm{H}_{7 / 2}$ and second excited ${ }^{6} \mathrm{H}_{9 / 2}$ state multiplets via crystal-field interaction here. Thus a $24 \times 24$ energy matrix is established. Substituting the free-ion and crystal-field parameters obtained from the optical spectra for the $\mathrm{Sm}^{3+} \mathrm{F}^{-}\left(\mathrm{C}_{4 \mathrm{v}}\right)$ center in $\mathrm{CaF}_{2}$ and $\mathrm{SrF}_{2}$ crystals (see Table 1) into the matrix and diagonalizing it, we can obtain that the wave function of the ground doublet of $\mathrm{CaF}_{2}: \mathrm{Sm}^{3+}$ is

$$
\begin{aligned}
\left|\Gamma_{6}\right\rangle \approx & \pm 0.9865|5 / 2, \pm 1 / 2\rangle-0.0413|7 / 2, \pm 1 / 2\rangle \\
& -0.0867|7 / 2, \mp 7 / 2\rangle \pm 0.0037|9 / 2, \pm 1 / 2\rangle \\
& \pm 0.1251|9 / 2, \mp 7 / 2\rangle \pm 0.0052|9 / 2, \pm 9 / 2\rangle,
\end{aligned}
$$

and that for $\mathrm{SrF}_{2}: \mathrm{Sm}^{3+}$ it is

$$
\begin{aligned}
\left|\Gamma_{6}\right\rangle \approx & \pm 0.9890|5 / 2, \pm 1 / 2\rangle-0.0326|7 / 2, \pm 1 / 2\rangle \\
& -0.0890|7 / 2, \mp 7 / 2\rangle \pm 0.0022|9 / 2, \pm 1 / 2\rangle \\
& \pm 0.1130|9 / 2, \mp 7 / 2\rangle \pm 0.0103|9 / 2, \pm 9 / 2\rangle .
\end{aligned}
$$

From the Zeeman interaction $H_{z}\left(=\mathrm{g}_{J} \mu_{\beta} \mathbf{H} \cdot \mathbf{J}\right.$, with the original meanings $[12,13])$ and hyperfine interaction $H_{\mathrm{hf}}\left(=P N_{J} \hat{N}\right.$, where $P$ is the dipolar hyperfine structure constant and $\mathrm{N}_{J}$ the diagonal matrix element for the ${ }^{2 S+1} L_{J}$ state [12]), we have the perturbation formulas of the EPR parameters for $4 \mathrm{f}^{n}$ ions as follows:

$g_{\|}=2 g_{J}\left\langle\Gamma \gamma\left|\hat{J}_{Z}\right| \Gamma \gamma\right\rangle, g_{\perp}=g_{J}\left\langle\Gamma \gamma\left|\hat{J}_{+}\right| \Gamma \gamma^{\prime}\right\rangle$,

$A_{\|}=2 P N_{\mathrm{J}}\left\langle\Gamma \gamma\left|\hat{N}_{\mathrm{Z}}\right| \Gamma \gamma\right\rangle, A_{\perp}=P N_{\mathrm{J}}\left\langle\Gamma \gamma\left|\hat{N}_{+}\right| \Gamma \gamma^{\prime}\right\rangle$.

Considering the covalence of the $\mathrm{Sm}^{3+}-\mathrm{F}^{-}$bond in both crystals, the orbital angular momentum $\hat{L}$ in the above formulas should be multiplied by an orbit reduction factor $k$. This factor, which is slightly smaller than 1 , depends on the covalence of the metal-ligand bond. The shorter the metal-ligand distance $R$, the stronger the covalence of this bond and so the smaller the factor $k$. This point can be confirmed by the following facts: (i) The covalence of the host crystal $\mathrm{CaF}_{2}$ is slightly stronger than that of the host crystal $\mathrm{SrF}_{2}$ [14]. (ii) The effect of pressure on the freeion parameters (Coulomb repulsion $F^{K}$ and spin-orbit coupling coefficient $\zeta_{4 \mathrm{f}}$ ) of the $\mathrm{Re}^{3+}$ ions in crystal $[15,16]$ suggests that these parameters decrease with decreasing metal-ligand distance and hence with increasing covalence of the bond. For $\mathrm{CaF}_{2}: \mathrm{Sm}^{3+}$, the above free-ion parameters are slightly smaller than those of $\mathrm{SrF}_{2}: \mathrm{Sm}^{3+}$ (see Table 1), and so its covalence is stronger. Thus we can reasonably assume for $\mathrm{CaF}_{2}: \mathrm{Sm}^{3+}, k \approx 0.976$ and for $\mathrm{SrF}_{2}: \mathrm{Sm}^{3+}, k \approx 0.980$. Applying (1) and (2), the factors $k$ and the free-ion values of $\mathrm{P}\left({ }^{147} \mathrm{Sm}\right) \approx-51.7(6) \times 10^{-4} \mathrm{~cm}^{-1}$ and $P$ $\left({ }^{149} \mathrm{Sm}\right) \approx-41.8(6) \times 10^{-4} \mathrm{~cm}^{-1}$ [12] to (3), the EPR parameters $g_{\|}, g_{\perp}, A_{\|}$and $A_{\perp}$ for the tetragonal $\mathrm{Sm}^{3+}$ centers in both crystals are calculated. The results are compared with the observed values in Table 2 .

\section{Conclusion and Discussion}

From Table 2, it can be seen that the calculated EPR parameters using the free- ion and crystal-field parameters obtained from the optical spectra of $\mathrm{Sm}^{3+}-\mathrm{F}^{-}$ $\left(\mathrm{C}_{4 \mathrm{v}}\right)$ centers in $\mathrm{CaF}_{2}$ and $\mathrm{SrF}_{2}$ crystals are consistent with the observed values given in [4-7]. So, the tetragonal EPR Sm${ }^{3+}$ center in $\mathrm{CaF}_{2}$ and $\mathrm{SrF}_{2}$ found in [4-7] rather than that in $[1,3]$ is the $\mathrm{Sm}^{3+}-\mathrm{F}^{-}$ $\left(\mathrm{C}_{4 \mathrm{v}}\right)$ center. The assignment can not be transformed by changing the orbit reduction factor $k$ because the factor $k$ affects slightly the calculated average value of $\bar{g}\left[=\left(g_{\|}+2 g_{\perp}\right) / 3\right]$ and $\bar{A}\left[=\left(A_{\|}+2 A_{\perp}\right) / 3\right]$, but it can not alter the sign of the anisotropy of the $\mathbf{g}$ factor and constant A (characterized by $\Delta g=g_{\|}-g_{\perp}$ and $\left.\Delta A=A_{\|}-A_{\perp}\right)$. So, the above assignment is reasonable 


\begin{tabular}{cccccccc}
\hline & $g_{\|}$ & $g_{\perp}$ & $A_{\|}\left({ }^{147} \mathrm{Sm}\right)$ & $A_{\perp}\left({ }^{147} \mathrm{Sm}\right)$ & $A_{\|}\left({ }^{149} \mathrm{Sm}\right)$ & $A_{\perp}\left({ }^{149} \mathrm{Sm}\right)$ \\
\hline & Cal. & 0.023 & 0.822 & 7.2 & $221 \pm 5$ & 6.1 & $180 \pm 4$ \\
$\mathrm{CaF}_{2}: \mathrm{Sm}^{3+}$ & Expt. [4-7] & $0 \pm 0.06$ & $0.823 \pm 0.003$ & $0 \pm 10$ & $230 \pm 5$ & $0 \pm 10$ & $190 \pm 5$ \\
& Expt. [1,3] & $0.907 \pm 0.010$ & $0.544 \pm 0.010$ & & & & \\
\hline $\mathrm{SrF}_{2}: \mathrm{Sm}^{3+}$ & Cal. & 0.027 & 0.824 & 7.3 & $225 \pm 5$ & 6.2 & $183 \pm 4$ \\
& Expt. [4-7] & $<0.1$ & $0.823 \pm 0.003$ & $0 \pm 10$ & $230 \pm 4$ & $0 \pm 10$ & $190 \pm 4$ \\
\hline
\end{tabular}

Table 2. The EPR $\mathbf{g}$ factors and hyperfine structure constants $A_{i}$ (in units of $10^{-4} \mathrm{~cm}^{-1}$ ) for the tetragonal $\mathrm{Sm}^{3+}$ center in $\mathrm{CaF}_{2}$ and $\mathrm{SrF}_{2}$ crystals.

and the $\mathbf{g}$ factors $g_{\|}, g_{\perp}$ and hyperfine structure constants $A_{\|}, A_{\perp}$ (for isotopes ${ }^{147} \mathrm{Sm}^{3+}$ and ${ }^{149} \mathrm{Sm}^{3+}$ ) observed in [4-7] for $\mathrm{Sm}^{3+}$-doped $\mathrm{CaF}_{2}$ and $\mathrm{SrF}_{2}$ can be

[1] M. J. Weber and R. W. Bierig, Phys. Rev. 134A, 1492 (1964).

[2] T. S. Chang and M. I. Cohen, J. Chem. Phys. 64, 5255 (1976).

[3] W. Low, Phys. Rev. 134A, 1479 (1964).

[4] A. A. Antinpin, I. N. Kurkin, L.D. Livanova, L.Z. Potvorova, and L. Ya. Shekun, Sov. Phys. Solid State 7, 1271 (1965).

[5] A. A. Antinpin, I. N. Kurkin, L.D. Livanova, L.Z. Potvorova, and L. Ya. Shekun, Sov. Phys. Tech. Phys. 11, 821 (1966).

[6] H. N. Evans and S. D. Mclaughlan, Phys. Lett. 23, 638 (1966).

[7] R. C. Newman and R. J. Woodward, J. Phys. C 7, L433 (1974).

[8] I. J. Ashburner, R. C. Newman, and S. D. Mclaughlan, Phys. Lett. 27A, 212 (1968). satisfactorily explained. The defect model of the tetragonal EPR $\mathrm{Sm}^{3+}$ center in $\mathrm{CaF}_{2}$ found in $[1,3]$ remains to be further studied.

[9] J. C. Wright and K. M. Cirillo-Penn, Radiat. Eff. Def. Solids, 119-121, 231 (1991).

[10] J.-P. R. Wells and R. J. Reeves, Phys. Rev. B61, 13593 (2000).

[11] M. Yamaga, M. Honda, J.-P. R. Wells, T. P. J. Han, and H. G. Gallagher, J. Phys.:Condens. Matter 12, 8727 (2000).

[12] A. Abragam and B. Bleanely, Electron Paramagnetic Resonance of Transition-Ions, Oxford University press, London 1970.

[13] I. A. Sorin and M. V. Vlasova, Electron Spin Resonance of Paramagnetic Crystals (Translated from Russian by P. Gluck ), Plenum Press, New York 1973.

[14] B. F. Levine, J. Chem. Phys. 59, 1463 (1973).

[15] C. Bungenstock, Th. Troster, and W. B. Holzapfel, Phys. Rev. B62, 7945 (2000).

[16] Y. R. Shen and W. B. Holzapfel, Phys. Rev. B52, 12618 (1995). 